\title{
miR-491-5p inhibits the proliferation and migration of A549 cells by FOXP4
}

\author{
FUYONG WU ${ }^{1}$, AIPING JI ${ }^{2}$, ZHENKUN ZHANG ${ }^{1},{\text { JINFANG } \mathrm{LI}^{1} \text { and PENGLONG LI }}^{3}$ \\ ${ }^{1}$ Third Department of Oncology and ${ }^{2}$ Second Department of Oncology, People's Hospital of \\ Shouguang, Shouguang, Shandong 262700; ${ }^{3}$ Department of Oncology, \\ Yantai Laiyang Central Hospital, Laiyang, Shandong 265200, P.R. China
}

Received July 15, 2018; Accepted March 11, 2021

DOI: $10.3892 /$ etm.2021.10054

\begin{abstract}
Aberrant expression of microRNAs (miRNAs/miRs) plays a key role in the development of non-small cell lung cancer (NSCLC). In the present study, lower miRNA (miR)-491-5p levels and a higher forkhead box P4 (FOXP4) mRNA level were observed in NSCLC tissues and cell lines, compared to adjacent tissues and the normal human lung epithelial cell line BEAS-2B, respectively. A549 cell proliferation and migration were inhibited upon transfection of miR-491-5p mimics compared to miR-negative control (NC) mimics. In addition, compared to miR-NC mimics, overexpression of miR-491-5p decreased FOXP4 expression, while downregulation of miR-491-5p increased FOXP4 expression in A549 cells. The dual luciferase assay confirmed that the 3'untranslated region of FOXP4 was a target for miR-491-5p in A549 cells. Moreover, compared with the control short hairpin (sh)RNA, there was lower expression levels of TGF- $\beta$ and its downstream targets (MMP-2 and MMP-9) in the FOXP4 shRNA group. Similarly, compared to miR-NC mimics, overexpression of miR-491-5p decreased MMP-2 and MMP-9 expression levels. In FOXP4-knockdown A549 cells, overexpression of miR-491-5p showed little effect on cell proliferation/migration. In A549 cells, overexpression of FOXP4 partially reversed the miR-491-5p mimics-induced inhibition on the cell proliferation and migration. These results may provide new insights into the role of miR-491-5p in NSCLC.
\end{abstract}

\section{Introduction}

Lung cancer is a major cancer type that threatens human life, with limited effective treatment approaches (1). Non-small cell lung cancer (NSCLC) accounts for $>80 \%$ of lung cancer cases

Correspondence to: Dr Penglong Li, Department of Oncology, Yantai Laiyang Central Hospital, 111 Changshan Road, Laiyang, Shandong 265200, P.R. China

E-mail: lipenglonglych@yeah.net

Key words: microRNA-491-5p, non-small cell lung cancer, cell proliferation, cell migration, forkhead box P4 and is relatively insensitive to chemotherapy or radiotherapy when compared with small cell lung cancer (2). Although much research has focused on the carcinogenesis behind and the development of anti-cancer drugs for NSCLC, the prognosis of patients with NSCLC remains poor due to drug resistance, metastasis and recurrence $(3,4)$. The current poor prognosis for patients with NSCLC emphasizes the urgent need for the discovery of new biomarkers and targets for the detection and treatment of the metastatic and NSCLC recurrence.

MicroRNAs (miRNAs/miRs) are 18-25-nucleotide long, non-coding RNAs that negatively regulate target gene expression by binding to the $3^{\prime}$ untranslated region (UTR) of target mRNAs (5). Deregulation of miRNAs is involved in both the initiation and development of various cancer types (6,7). A specific miRNA may function as an oncogene or a tumor suppressor depending on the target mRNAs in different cellular contexts (8). A number of miRNAs have been discovered to drive or inhibit the progression of NSCLC by targeting critical genes in cancer-related pathways (9-11). Among them, the expression of several miRNAs is considered to be a diagnostic or prognostic biomarker for patients with NSCLC $(12,13)$. For example, miRNA-7 serves as a tumor suppressor in NSCLC through inhibiting the expression of paired box 6 and protein tyrosine kinase $2(14,15)$. Comparison of miR-7 expression levels in patients with NSCLC indicates that the miR-7 expression levels are a marker of the therapeutic effects of gefitinib (16). Bioinformatics analysis of NSCLC microarray data has revealed that miR-491-5p is a potential regulator of the development of NSCLC (17). However, the molecular mechanism of action behind how miR-491-5p regulates NSCLC is unknown.

Forkhead box (FOX)P4 is a member of the FOX transcription factor family (18). Overexpression of FOXP4 is observed in hepatocellular carcinoma and osteosarcoma, when compared with normal tissues $(19,20)$. It has previously been shown that FOXP4 is a target of miR-138 and that silencing FOXP4 reduces cell proliferation and cell invasion capacity in NSCLC cells (21).

The present study aimed to investigate the effects of miR-491-5p on proliferation and migration of A549 cells, and to determine whether miR-491-5p directly interacted with FOXP4. It was found that miR-491-5p was significantly downregulated in NSCLC tissue. miR-491-5p overexpression 
inhibited the cell proliferation/migration of NSCLC cells. Collectively, the present findings suggested a tumor suppressor role of miR-491-5p in NSCLC and may provide a potential target for the treatment of NSCLC.

\section{Materials and methods}

Clinical samples. Between January 2015 and June 2016, 40 patients (25 males, 15 females; age, 45-70 years) with NSCLC from Yantai Laiyang Central Hospital (Laiyang, China) were enrolled in the present study. Patients who had received preoperative anti-tumor therapy were excluded from the present study. Written consent was received from all participants. Tumor tissues and paired adjacent normal tissues were collected and immediately stored at $-80^{\circ} \mathrm{C}$ for subsequent RNA extraction. All experiments were approved and conducted under the supervision of the ethics committee of Yantai Laiyang Central Hospital.

Cell culture. The human lung epithelial cell line BEAS-2B, and human NSCLC cell lines including A549, H157 and H1650, were purchased from the American Type Culture Collection. All cell lines were cultured in DMEM (Gibco; Thermo Fisher Scientific, Inc.) supplemented with 10\% FBS (HyClone; GE Healthcare Life Sciences) in a $37^{\circ} \mathrm{C}$ incubator with $5 \% \mathrm{CO}_{2}$.

Transfection of miRNA mimics and miRNA inhibitor. miR-491-5p mimics (5'-AGUGGGGAACCCUUCCCAUGA GG-3'), miR-NC mimics (5'-AGAAGCUGUUCCAAGGUG GGCC-3'), miR-491-5p inhibitor (5'-CCUCAUGGAAGG GUUCCCCACU-3') and miR-NC inhibitor (5'-GAACAUCCA GGGUCCCGUUCCU-3') were purchased from Shanghai GenePharma Co., Ltd. For cell transfection, miRNA mimics and miRNA inhibitor were transfected into A549 cells using Lipofectamine ${ }^{\circledR} 2000$ (Invitrogen; Thermo Fisher Scientific, Inc.) according to the manufacturer's protocol. Briefly, the cells $\left(8 \times 10^{5}\right.$ cells/well) were seeded into 6 -well plates. $50 \mathrm{nM}$ miRNA mimics or miRNA inhibitor was mixed with Lipofectamine 2000 in Opti-MEM (Invitrogen; Thermo Fisher Scientific, Inc.) and placed at room temperature for $10 \mathrm{~min}$. Subsequently, the mixture was added into each well. Following $6 \mathrm{~h}$ of transfection at $37^{\circ} \mathrm{C}$, the culture medium was replaced with fresh medium and cells were cultured for another $24 \mathrm{~h}$ at $37^{\circ} \mathrm{C}$ before being subjected to the following experiments.

Cell proliferation assay. The cell proliferation of A549 cells was detected using a Cell Counting Kit-8 (CCK-8; Dojindo Molecular Technologies, Inc.) according to manufacturer's protocol. In brief, transfected cells were seeded in 96-well plates at a density of $2 \times 10^{4}$ cells/well. At the various time points $(0,24,48$ and $72 \mathrm{~h}), 10 \mu \mathrm{lCCK}-8$ solution was added into each well and cultured at $37^{\circ} \mathrm{C}$ for another $2 \mathrm{~h}$. Subsequently, the medium containing CCK- 8 was transferred into another 96-well plate and the absorbance at $450 \mathrm{~nm}$ was detected using a microplate reader (Bio-Rad Laboratories, Inc.).

Cell migration assay. The migration of A549 cells was measured using wound healing assays. Cells $\left(\sim 5 \times 10^{5}\right.$ cells/well $)$ were grown in 6-well plates and cultured to $100 \%$ confluence. A wound area was made by scratching the central area of each well using a $10 \mu \mathrm{l}$ pipette. The cells were cultured with serum-free DMEM to avoid cell proliferation. IncuCyte ZOOM (Essen BioScience) was used to capture the images of cell migration with a confocal microscope (x200 magnification). The wound areas were analyzed using Image Pro Plus (version 6.0; Media Cybernetics, Inc.) and the wound closure percentage was calculated as a reflection of migratory ability. The following equation was used: Migration rate $=\left(\right.$ Wound $_{30} /$ Wound $\left._{0}\right) \times 100 \%$.

Western blot analysis. Protein lysates of A549 cells were collected using RIPA lysis buffer (Sigma-Aldrich; Merck $\mathrm{KGaA}$ ) according to the manufacturer's protocol. Antibodies against TGF- $\beta$ (cat. no. 3711; 1:1,000), MMP-2 (cat. no. 40994; 1:1,000) and MMP-9 (cat. no. 13667; 1:1,000) were purchased from Cell Signaling Technology, Inc. FOXP4 antibodies (cat. no. ab119404; 1:1,000) were purchased from Abcam. The GAPDH (cat. no. G8795; 1:10,000) antibody was purchased from Sigma-Aldrich; Merck KGaA. HRP-conjugated secondary antibodies against rabbit (cat. no. SA00001-2; $1: 10,000)$ and mouse (cat. no. SA00001-1; 1:10,000) were obtained from Proteintech Group, Inc. Proteins were quantified using a BCA assay kit (Pierce; Thermo Fisher Scientific, Inc.). Lysates containing $20 \mu \mathrm{g}$ protein were loaded and separated on an $8 \%$ SDS-PAGE gel. The proteins were transferred onto a PVDF membrane and then blocked in 5\% non-fat milk at room temperature for $2 \mathrm{~h}$. Subsequently, the membrane was incubated in the primary antibodies overnight at $4{ }^{\circ} \mathrm{C}$, followed by incubation in the secondary antibody at room temperature for another $1 \mathrm{~h}$. The membrane was developed using ECL Western Blot Substrate (Pierce; Thermo Fisher Scientific, Inc.). The intensity of the bands was analyzed using ImageJ version 1.8.0 (National Institutes of Health).

RNA extraction and reverse transcription-quantitative PCR (RT-qPCR). Total RNA was extracted from tissues and cells using TRIzol ${ }^{\boxplus}$ reagent (Invitrogen; Thermo Fisher Scientific, Inc.). For the detection of the expression levels of miR-491-5p, RNA was reverse transcribed using a Mir-X miRNA First-Strand Synthesis kit (Takara Bio, Inc.) according to the manufacturer's protocol. miR-491-5p expression was conducted using SYBR Premix Ex Taq II (Takara Bio, Inc.). U6 served as an internal control for miR-491-5p. For the detection of mRNA levels of FOXP4, MMP-2 and MMP-9, RNA was reverse transcribed into cDNA using PrimeScript RT Master Mix (Takara Bio, Inc.). Gene expression was conducted using SYBR Premix Ex Taq II (Takara Bio, Inc.). GAPDH served as an internal control for the mRNA of FOXP4, MMP-2 and MMP-9. The thermocycling parameters were as follows: Initial denaturation at $95^{\circ} \mathrm{C}$ for $2 \mathrm{~min}, 40$ cycles of $95^{\circ} \mathrm{C}$ for $15 \mathrm{sec}$ and $64^{\circ} \mathrm{C}$ for $30 \mathrm{sec}$. The primer sequences were as follows: miR-491-5p forward, 5'-GGAGTGGGGAAC CCTTCC-3' and reverse, 5'-GTGCAGGGTCCGAGGT-3'; U6 forward, 5'-GTGCTCGCTTCGGCAGCACAT-3' and reverse, 5'-AATATGGAACGCTTCACGAAT-3'; FOXP4 forward, 5'-GACAGCCTACTGTGCTCACAT-3' and reverse, 5'-TTG CACTCTCCGTGTCCGTA-3'; MMP-2 forward, 5'-TACAGG ATCATTGGCTACACACC-3' and reverse, 5'-GGTCACATC 
GCTCCAGACT-3'; MMP-9 forward, 5'-TGTACCGCTATG GTTACACTCG-3' and reverse, 5'-GGCAGGGACAGTTGC TTCT-3'; and GAPDH forward, 5'-GGAGCGAGATCCCTC CAAAA-3' and reverse, 5'-GGCTGTTGTCATACTTCTCAT GG-3'. The relative expression levels were calculated using the $2^{-\Delta \Delta \mathrm{CC}}$ method (22).

Lentivirus system. The FOXP4 knockdown A549 cell line (A549-FOXP4 shRNA) and control A549 cell line (A549-control shRNA) were established using a lentivirus system. Briefly, shRNA sequences targeting FOXP4 or non-specific sequences were synthesized and cloned into the pLKO.1 plasmid (Shaanxi YouBio Technology Co., Ltd.). The pLKO.1-FOXP4 shRNA or pLKO.1-control shRNA was co-transfected with packaging plasmids into 293 cells to obtain the virus. The virus was collected $48 \mathrm{~h}$ later and transfected into target cells using Lipofectamine ${ }^{\circledR} 2000$ (Invitrogen; Thermo Fisher Scientific, Inc.) at $37^{\circ} \mathrm{C}$ for $48 \mathrm{~h}$. The cells that were successfully infected with virus were screened by exposure to puromycin $(10 \mu \mathrm{M}$; Sigma-Aldrich; Thermo Fisher Scientific, Inc.) for $72 \mathrm{~h}$.

Dual luciferase reporter assay. FOXP4 was predicted to be targeted by miR-491-5p using TargetScan (www.targetscan. org). The 3'UTR of FOXP4 mRNA was amplified from cDNA of BEAS-2B cells and ligated into the pGL3 plasmid. The amplification of the FOXP4 3'UTR was achieved using PrimeSTAR Max DNA polymerase (Takara Bio, Inc.) with two primers. The primer sequences were: FOXP4 3'UTR forward, 5'-GGG CCTGTAGTGACCGGCAG-3' and FOXP4 3'UTR reverse, 5'-AATTGTTTTTATTGCATTGCATTGT-3'. Two site mutations were introduced into pGL3-FOXP4 3'UTR-wild-type (WT) to construct the pGL3-FOXP4 3'UTR-mutant (Mut) using a QuickChange Site-Directed Mutagenesis kit (Stratagene; Agilent Technologies, Inc.) following the manufacturer's protocol. pGL3-FOXP4 3'UTR-WT and pGL3-FOXP4 3'UTR-Mut were co-transfected into A549 cells with miR-NC mimics or miR-491-5p mimics using Lipofectamine ${ }^{\circledR} 2000$ (Invitrogen; Thermo Fisher Scientific, Inc.). After 48 h, the luciferase activity of each well was calculated using a Dual Luciferase Reporter System (Promega Corporation). The firefly luciferase activity was normalized to Renilla luciferase activity.

Overexpression of FOXP4. Full-length FOXP4 was amplified from A549 cDNA using PrimeSTAR Max DNA polymerase (Takara Bio, Inc.) and inserted into the pcDNA3.1 plasmid (YouBio). The primer sequences were: FOXP4 forward, 5'-GCTTGGTACCGAATGATGGTGGAATCT GCCTCG-3' and FOXP4 reverse, 5'-CCGCTCGAGGGACAG TTCTTCTCCCGGCA-3'. For overexpression of FOXP4, $2 \mu \mathrm{g}$ pcDNA3.1-FOXP4 was mixed with Lipofectamine ${ }^{\circledR} 2000$ in serum-free DMEM for $15 \mathrm{~min}$ and then added into cultured cells $\left(2 \times 10^{6}\right.$ A549 cells/well in 6-well plates) at room temperature. The cells were transfected for $48 \mathrm{~h}$ at $37^{\circ} \mathrm{C}$ before being subjected to further experiments.

Statistical analysis. All data were analyzed using GraphPad Prism 6 (GraphPad Software, Inc.) and are presented as the mean \pm SD deviation of 3 independent repeats. The results in Figs. $1 \mathrm{~A}$ and $\mathrm{B}$, and $2 \mathrm{~B}$ and $\mathrm{E}$ were analyzed using paired Student's t-test, while the results in Fig. 1C were analyzed using Pearson correlation analysis. The data from Fig. 2A were analyzed using a one-way ANOVA and post-hoc Tukey's tests. The comparisons between two groups in the remaining figures were conducted using unpaired Student's t-tests. Differences among more than two groups were calculated using one-way ANOVAs followed by post hoc Student-Newman-Keul's or Tukey's tests. $\mathrm{P}<0.05$ was considered to indicate a statistically significant difference.

\section{Results}

Lower miR-491-5p and higher FOXP4 expression is found in NSCLC tissues. RT-qPCR was first used to detect the miR-491-5p expression levels and FOXP4 mRNA expression levels in tumor tissues and matched normal tissues from 40 patients with NSCLC. Compared with the normal tissues, there was significantly lower miR-491-5p levels and higher FOXP4 mRNA levels in the tumor tissues (Fig. 1A and B). Pearson correlation analysis confirmed that miR-491-5p levels were negatively associated with FOXP4 mRNA levels in NSCLC tumor tissues (Fig. 1C).

miR-491-5p mimics inhibits A549 cell proliferation/migration. RT-qPCR was performed to detect the miR-491-5p expression levels in a normal lung cell line (BEAS-2B) and NSCLC cell lines (A549, H157 and H1650). Compared with BEAS-2B cells, there was significantly lower miR-491-5p expression in all NSCLC cell lines (Fig. 2A). A549 cells were used for the subsequent experiments as they had the lowest miR-491-5p expression levels among the three cell lines. To investigate the function of miR-491-5p in NSCLC, miR-491-5p mimics were transfected into A549 cells. Significantly increased miR-491-5p levels were observed in the miR-491-5p mimics group compared to the miR-NC mimics group (Fig. 2B). In comparison with the miR-NC mimics group, miR-491-5p mimics significantly decreased A549 cell proliferation (Fig. 2C). In addition, compared with the miR-NC mimics group, miR-491-5p mimics reduced the wound closure areas in A549 cells, indicating that miR-491-5p inhibited the cell migratory ability of A549 cells (Fig. 2D and E).

miR-491-5p targets and decreases FOXP4 expression levels in A549 cells. To validate the regulatory effect of miR-491-5p on FOXP4 expression, miR-491-5p was overexpressed in A549 cells and the FOXP4 expression levels were analyzed. Consistent with their association in NSCLC tumor tissues, compared with the miR-NC mimics group, miR-491-5p mimics significantly decreased FOXP4 at the mRNA and protein expression levels in A549 cells (Fig. 3A-C). The expression levels of miR-491-5p significantly decreased in cells transfected with miR-491-5p inhibitor compared with the control transfection (Fig. S1). Downregulation of miR-491-5p increased FOXP4 mRNA and protein levels in A549 cells (Fig. 3D-F). A putative binding site between miR-491-5p and the FOXP4 3'UTR sequence was predicted using TargetScan software (Fig. 3G). To determine whether FOXP4 was a direct target of miR-491-5p in NSCLC cells, a dual luciferase assay was used to investigate the binding sites between miR-491-5p and FOXP4 mRNA. Compared with 

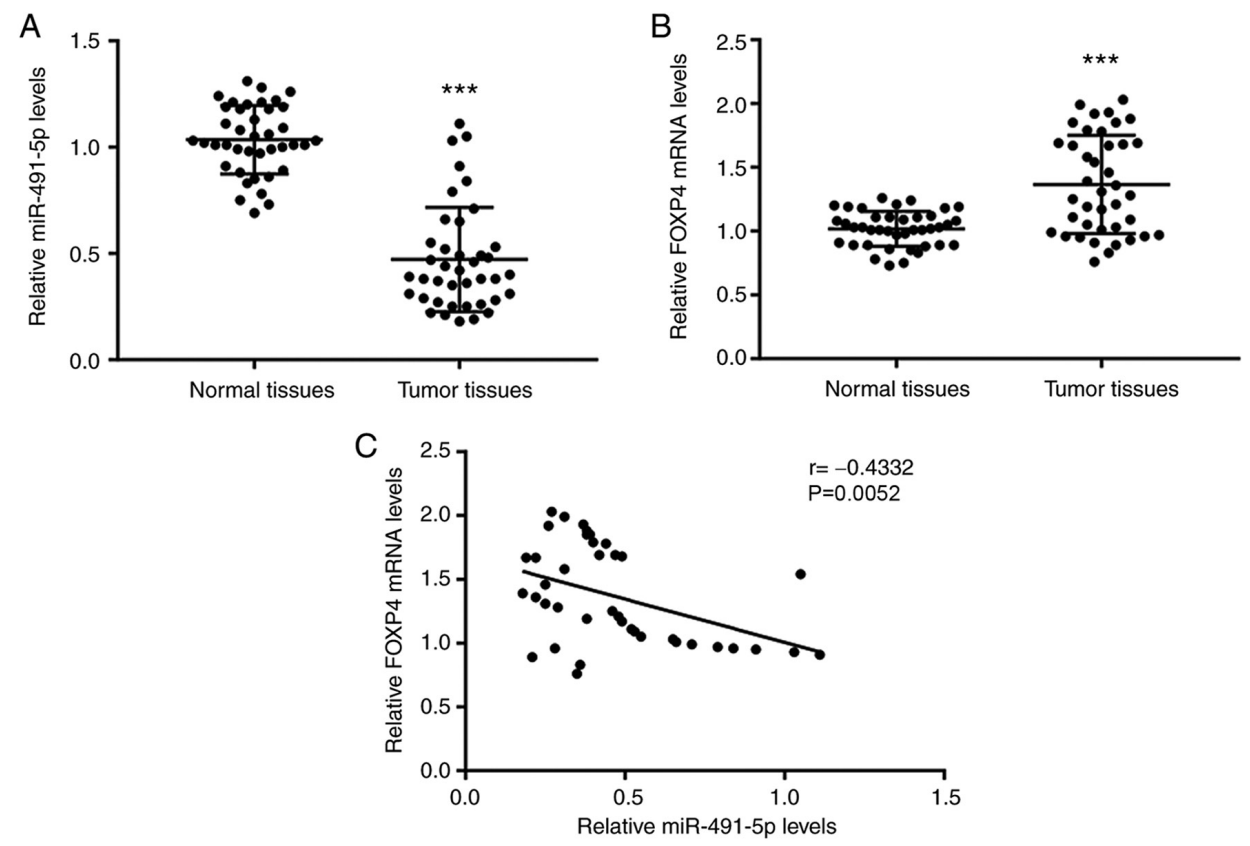

Figure 1. Aberrant expression of miR-491-5p and FOXP4 in NSCLC tissues. Compared with the adjacent normal tissues, (A) miR-491-5p expression levels were decreased and (B) FOXP4 mRNA was increased in tumor tissues from 40 patients with NSCLC. (C) Pearson correlation analysis showed a significant negative correlation between miR-491-5p expression levels and FOXP4 mRNA expression levels. ${ }^{* * *} \mathrm{P}<0.001$ vs. normal tissues. FOXP4, forkhead box P4; miR, microRNA; NSCLC, non-small cell lung cancer.
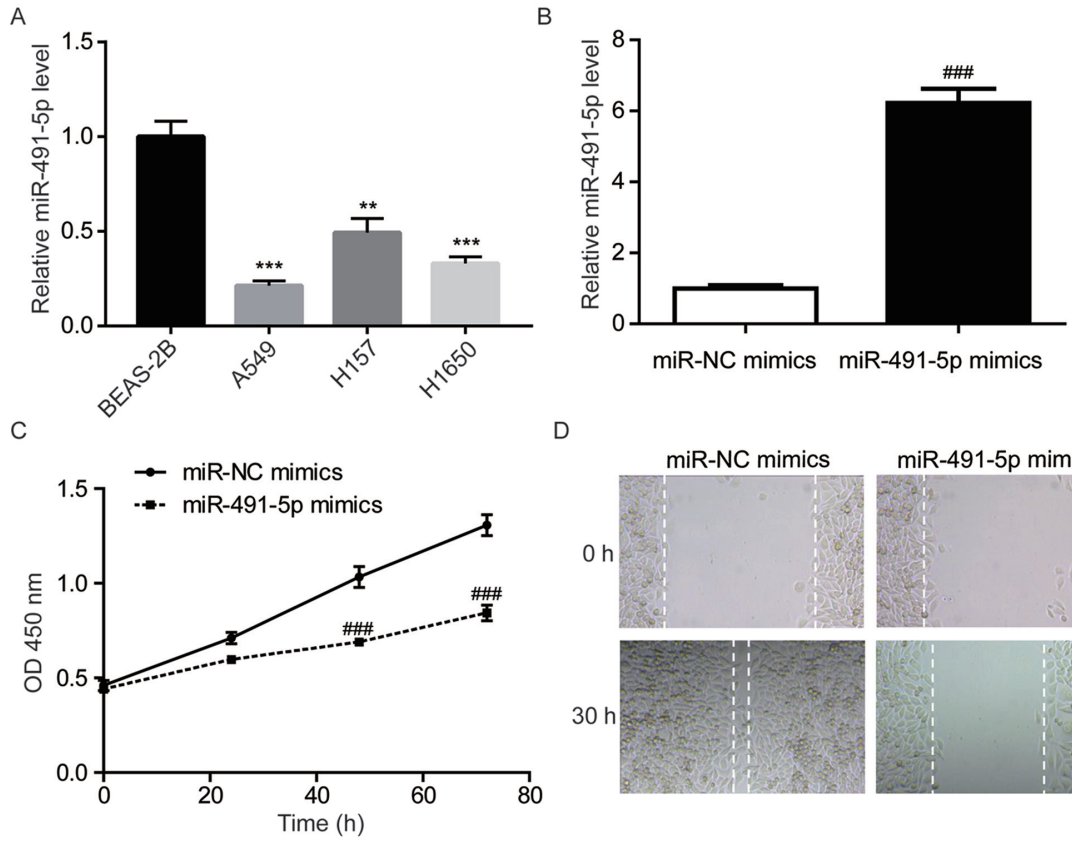

D
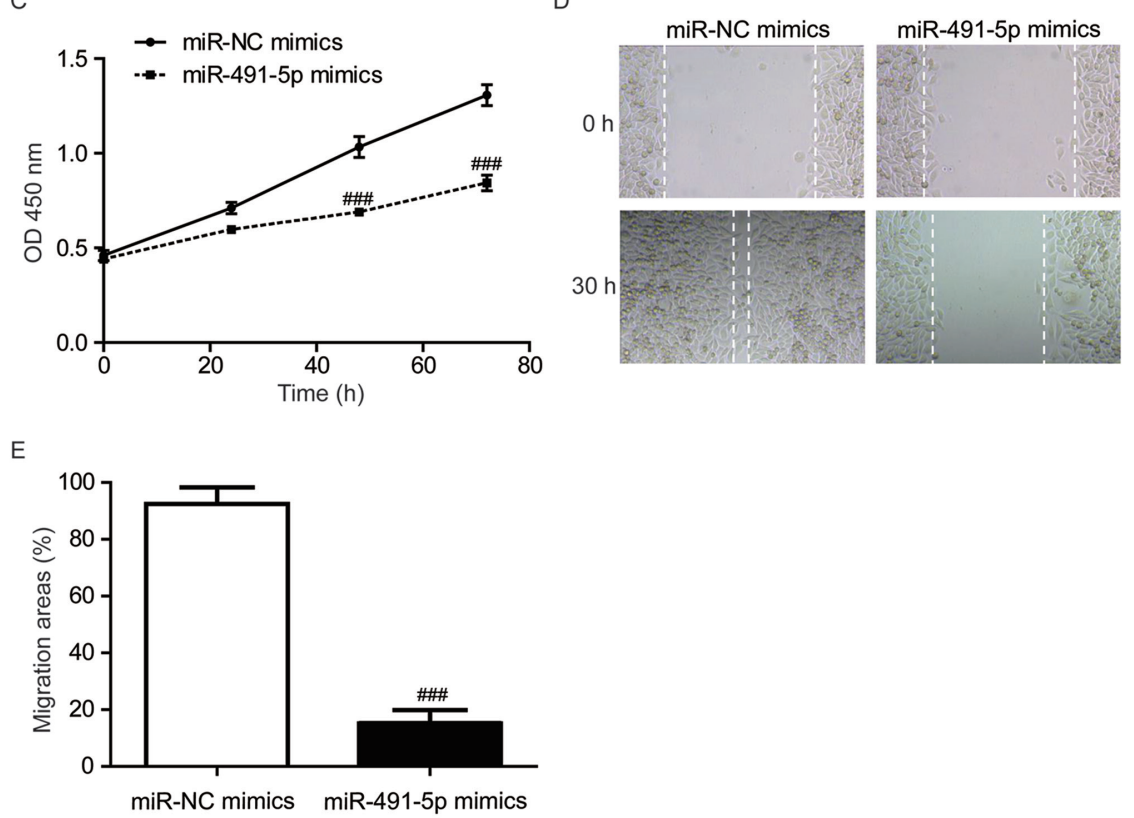

Figure 2. miR-491-5p inhibits A549 cell proliferation and migration. (A) Compared with BEAS-2B cells, miR-491-5p expression levels were decreased in non-small cell lung cancer cell lines (A549, H157 and H1650). (B) Compared with the miR-NC mimics group, miR-491-5p mimics significantly increased miR-491-5p expression levels in A549 cells. Compared with the miR-NC mimics group, miR-491-5p mimics significantly inhibited (C) cell proliferative and (D and E) cell migratory abilities of A549 cells. ${ }^{* *} \mathrm{P}<0.01$ and ${ }^{* * *} \mathrm{P}<0.001$ vs. BEAS-2B; ${ }^{\# \# /} \mathrm{P}<0.001$ vs. miR-NC mimics. miR, microRNA; NC, negative control; OD, optical density. 


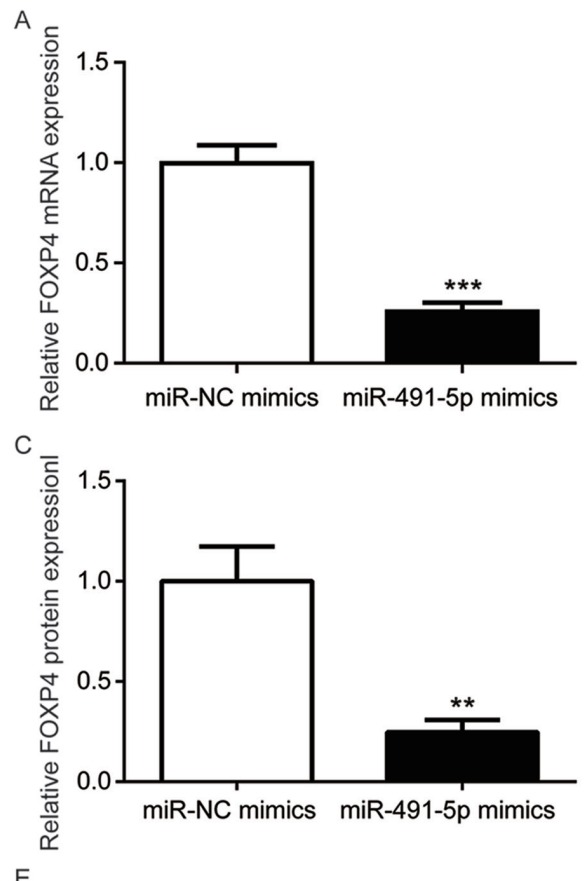

B

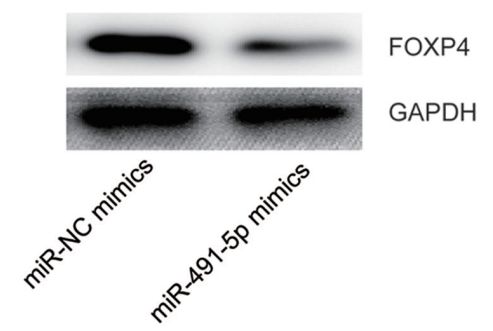

D

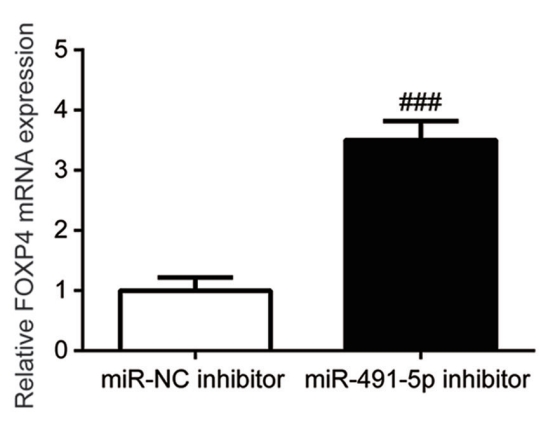

$\mathrm{E}$

$\mathrm{F}$

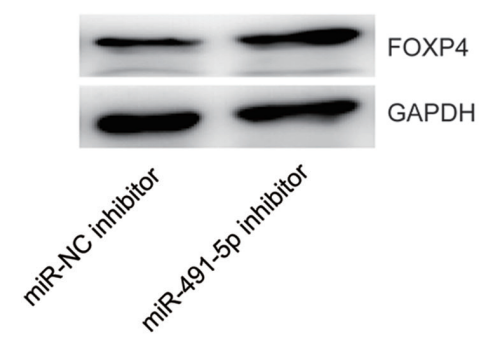

G

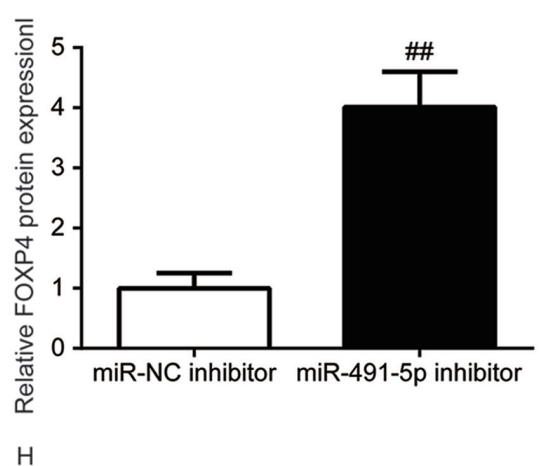

FOXP4-WT 5'-UAUUUCCCAAGAACCCCCCACAU-3'

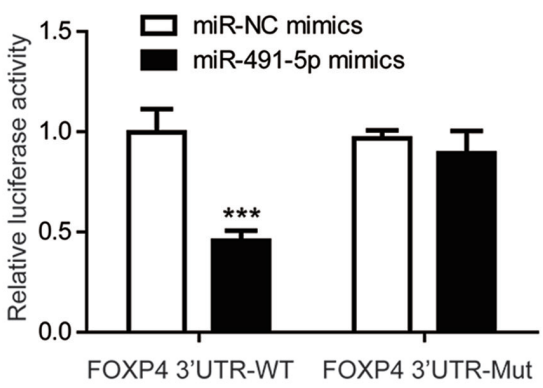

Figure 3. miR-491-5p binds to the 3'UTR of FOXP4 in A549 cells. Compared with miR-NC mimics group, miR-491-5p mimics reduces FOXP4 (A) mRNA and (B and C) protein expression levels in A549 cells. (D) Compared with the miR-NC inhibitor group, miR-491-5p inhibitor increases FOXP4 (D) mRNA and (E and F) protein expression levels in A549 cells. (G) The putative binding site between miR-491-5p and the FOXP4 3'UTR, as well as the constructed FOXP4 3'UTR-Mut are shown. (H) Compared with the miR-NC mimics group, miR-491-5p mimics led to a reduction in luciferase activity of A549 cells transfected with pGL3-FOXP4 3 'UTR-WT $(\mathrm{H}) .{ }^{* *} \mathrm{P}<0.01,{ }^{* * *} \mathrm{P}<0.001$ vs. miR-NC mimics; ${ }^{\# \#} \mathrm{P}<0.01$ and ${ }^{\# \# \#} \mathrm{P}<0.001$ vs. miR-NC inhibitor. FOXP4, forkhead box P4; miR, microRNA; Mut, mutant; NC, negative control; UTR, untranslated region; WT, wild-type.

the miR-NC mimics group, miR-491-5p mimics significantly decreased the luciferase activity of A549 cells transfected with FOXP4 3'UTR-WT but not FOXP4 3'UTR-Mut, indicating a regulatory relationship between miR-491-5p and FOXP4 in A549 cells (Fig. 3H).

FOXP4 knockdown suppresses TGF- $\beta$ signaling in A549 cells. FOXP4 regulates target gene expression through its interaction with FOXP1/FOXP2 (23). FOXP2 stimulates cancer cell migration by promoting TGF- $\beta$ expression (24). A FOXP4-knockdown model was constructed in A549 cells using a lentivirus system to study the function of miR-491-5p. As shown in Fig. 4A and B, FOXP4 expression was greatly reduced in FOXP4-knockdown A549 cells compared with control shRNA A549 cells. In FOXP4-knockdown A549 cells, TGF- $\beta$ protein expression levels were significantly reduced compared with the control shRNA A549 cells (Fig. 4A and B). MMP-2 and MMP-9 are downstream target genes of TGF- $\beta$ (25). It was found that in the FOXP4-knockdown group, there were significantly reduced mRNA and protein expression levels of MMP-2 and MMP-9 in comparison with the control shRNA group (Fig. 4C-E). Notably, overexpression 
A

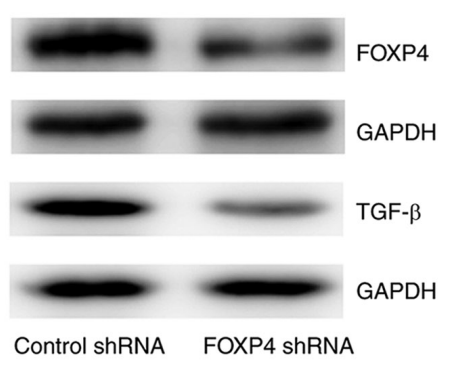

C

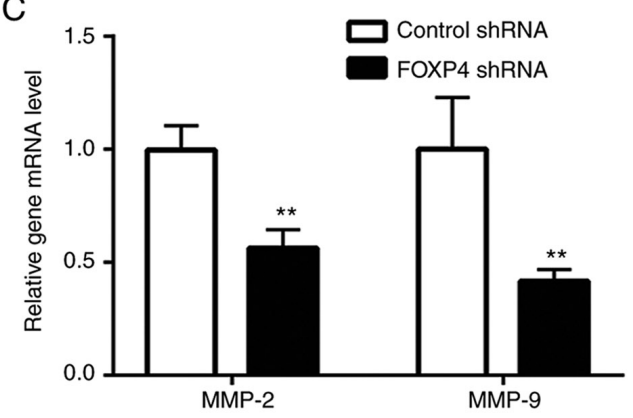

E

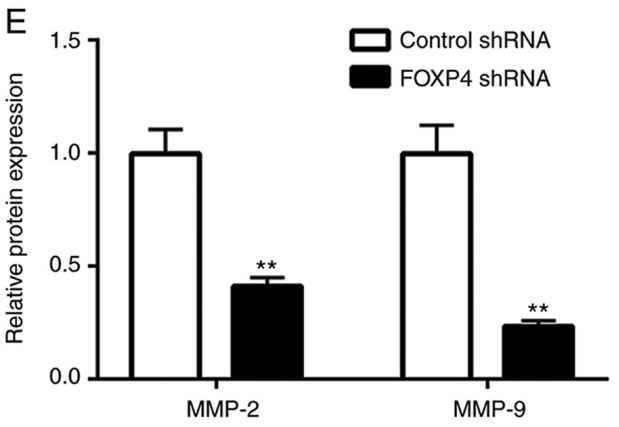

G

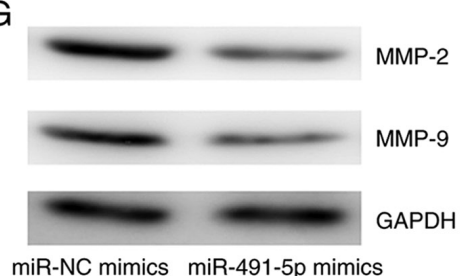

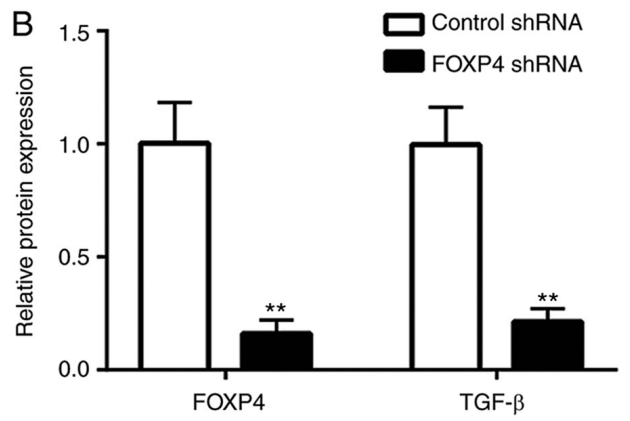

D

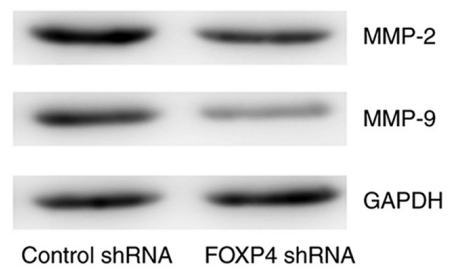

F
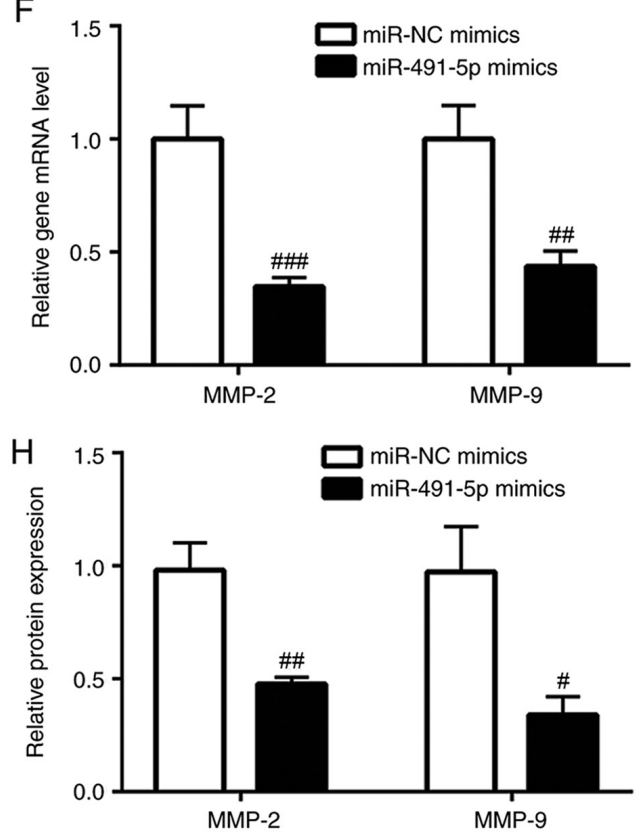

Figure 4. Knockdown of FOXP4 inactivates the TGF- $\beta$ signaling pathway and reduces MMP-2 and MMP-9 expression levels in A549 cells. (A and B) Compared with the control shRNA group, lentivirus mediated knockdown of FOXP4 significantly reduced FOXP4 and TGF- $\beta$ protein expression levels in A549 cells. Compared with A549-control shRNA, (C) mRNA and (D and E) protein expression levels of MMP-2 and MMP-9 were reduced in A549-FOXP4 shRNA cells. Overexpression of miR-491-5p decreased (F) MMP-2 and MMP-9 mRNA and (G and H) protein expression levels in A549 cells. ** $<0.01$ vs. Control shRNA; ${ }^{\#} \mathrm{P}<0.05,{ }^{\# \#} \mathrm{P}<0.01$ and ${ }^{\# \# \#} \mathrm{P}<0.001$ vs. miR-NC mimics. FOXP4, forkhead box P4; miR, microRNA; NC, negative control; shRNA, short hairpin RNA.

of miR-491-5p also significantly decreased MMP-2 and MMP-9 expression levels in A549 cells, in comparison with the miR-NC mimics group (Fig. 4F-H), suggesting the existence of an miR-491-5p/FOXP4/TGF- $\beta$ axis in NSCLC cells.

miR-491-5p mimics do not affect cell proliferation/migration in FOXP4-knockdown A549 cells. Subsequently, the present study sought to investigate whether FOXP4 was indispensable to miR-491-5p on its regulation of cell proliferation/migration. In FOXP4-knockdown A549 cells, miR-491-5p mimics exerted a slight, but non-significant effect on cell proliferation/migration compared to the miR-NC mimics group (Fig. 5A-C).
FOXP4 is pivotal for the function of miR-491-5p in NSCLC cells. The pcDNA3.1-FOXP4 vector was constructed to overexpress FOXP4 in A549 cells, which was found to effectively increase FOXP4 protein expression levels (Fig. 6A and B). It was observed that the overexpression of FOXP4 reversed the inhibition of cell proliferation induced by miR-491-5p mimics in A549 cells (Fig. 6C). In addition, FOXP4 elevation partially reversed the inhibition of cell migration induced by miR-491-5p mimics in A549 cells (Fig. 6D and E). The data suggested that miR-491-5p partially relied on the regulation of FOXP4 to control NSCLC cell proliferation and migration. 


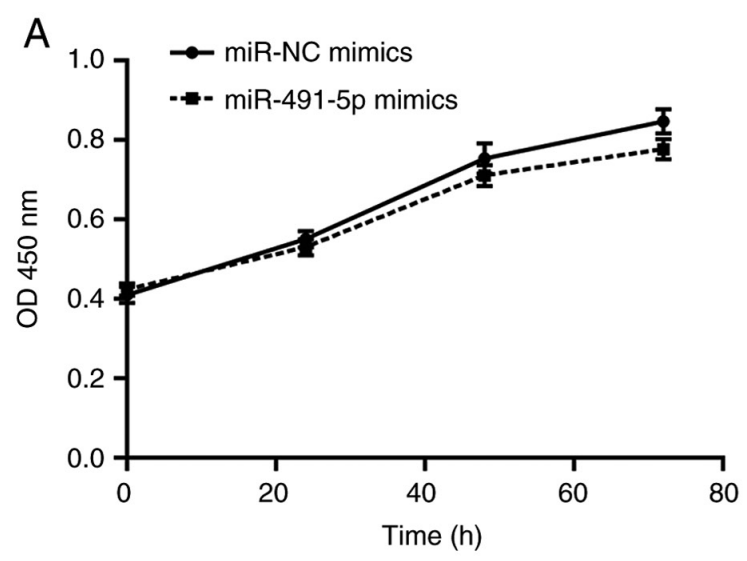

B
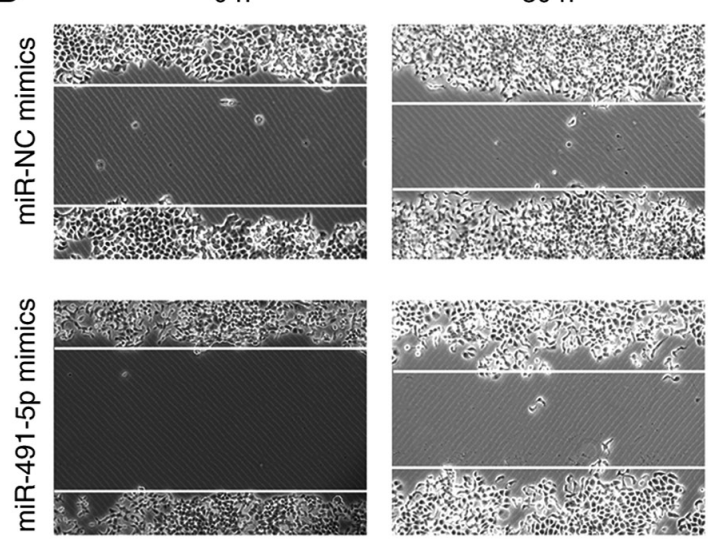

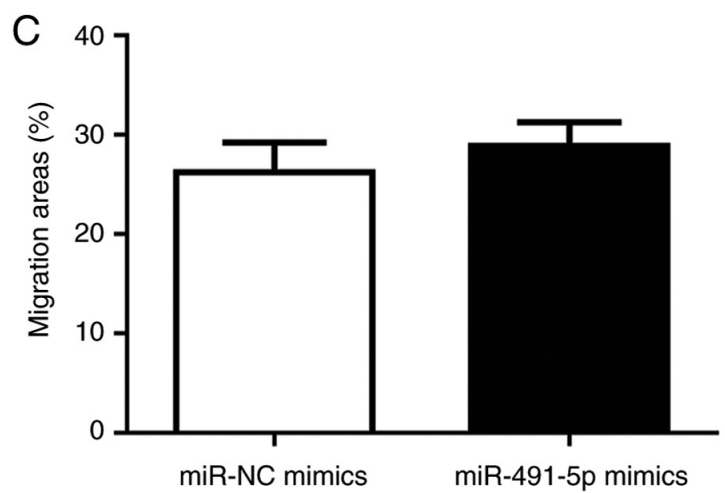

Figure 5. Overexpression of miR-491-5p does not inhibit cell proliferation or cell migration of A549-FOXP4 shRNA cells. In A549-FOXP4 shRNA cells, overexpression of miR-491-5p showed no obvious (A) anti-proliferative effect or (B and C) anti-migratory effect, compared with transfection of miR-NC mimics (magnification, x200). miR, microRNA; NC, negative control; OD, optical density.

\section{Discussion}

Currently, the prognosis for patients with NSCLC is not satisfactory and attempts to improve the clinical outcome have largely relied on the discovery of novel targets and biomarkers. miRNAs have captured the attention of researchers in terms of their function during the initiation and development of NSCLC $(2,6)$. The present study showed that miR-491-5p inhibited NSCLC cell proliferation/migration by targeting FOXP4.

Accumulating evidence suggests that miR-491-5p is a tumor suppressor in various types of cancer, including breast cancer, prostate cancer and gastric cancer (26-28). The present study found that miR-491-5p levels were decreased in tumor tissues compared with matched normal tissues from patients with NSCLC. In addition, enhanced expression levels of miR-491-5p led to a reduction of cell proliferation/migration in A549 cells. These data are consistent with a recent study which showed that miR-491-5p is a tumor suppressor in NSCLC (29). miR-491-5p suppresses cancer progression by targeting various oncogenes according to the context within the type of cell $(30,31)$.

FOXP4 promotes NSCLC progression by accelerating cell proliferation and enhancing the invasive ability of NSCLC cells (21). In the present study, miR-491-5p mimics led to a decrease in FOXP4 expression. miR-491-5p inhibitor, on the contrary, increased FOXP4 expression in A549 cells. FOXP4 has been validated to be a target gene of miR-491-5p in human osteosarcoma (19). Using a dual luciferase reporter assay, the present study showed that miR-491-5p could directly bind to the 3'UTR of FOXP4 mRNA in A549 cells.

Previous studies have indicated that miR-491-5p targets MMP-9 and insulin-like growth factor 2 mRNA-binding protein 1 to suppress cell proliferation and cell migration in non-small cell lung cancer $(29,32)$. MMP-2 and MMP-9 are key regulators of metastasis in cancer cells (33). The TGF- $\beta$ signaling pathway plays a critical role in mediating metastasis, partly by promoting the expression of MMP-2 and MMP-9 (34). In the current study, it was discovered that knockdown of FOXP4 reduced the TGF- $\beta$ protein expression levels and decreased the expression of MMP-2 and MMP-9 in A549 cells. Meanwhile, in FOXP4-knockdown A549 cells, transfection of miR-491-5p mimics did not inhibit cell proliferation or cell migration. Additionally, overexpression of FOXP4 was able to partially reverse the miR-491-5p mimics-induced inhibition of cell proliferation/migration in A549 cells. Thus, these data suggested that the inhibitory effect of miR-491-5p on cell proliferation and migration mainly relies on its regulation of FOXP4 in NSCLC cells.

In conclusion, the present study revealed a tumor suppressor role for miR-491-5p in NSCLC. Mechanistically, miR-491-5p may have inhibited the cell proliferation/migration of A549 cells, as well as the activation of the TGF- $\beta$ signaling pathway by directly binding to the 3'UTR of FOXP4 mRNA. The present findings enhance the current understanding of the role of miR-491-5p in NSCLC, 
A

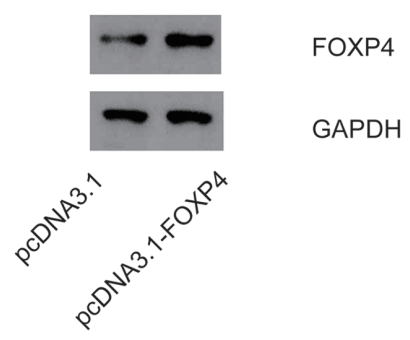

C

- miR-NC mimics + pcDNA3.1

miR-491-5p mimics + pcDNA3.1

-.. miR-491-5p mimics + pcDNA3.1-FOXP4

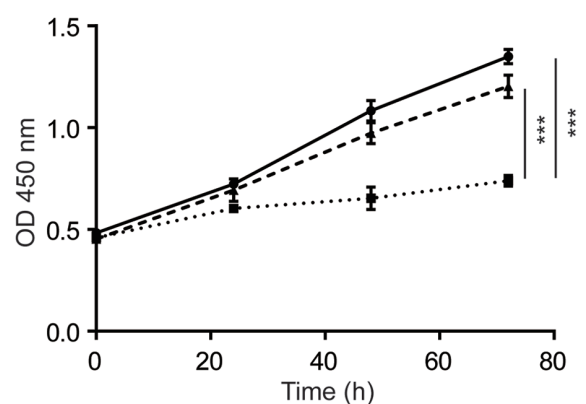

E

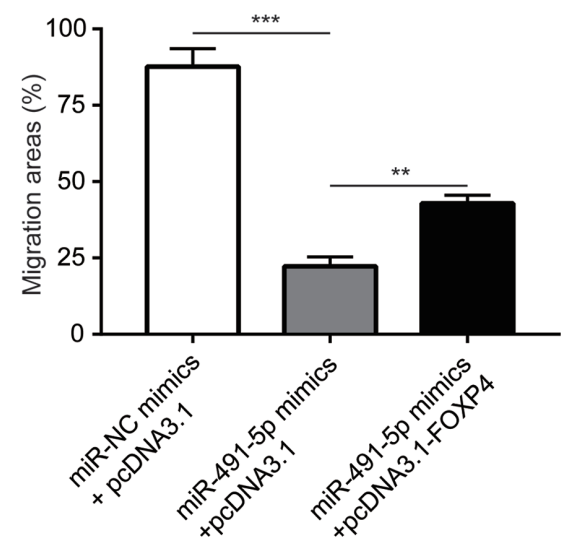

B

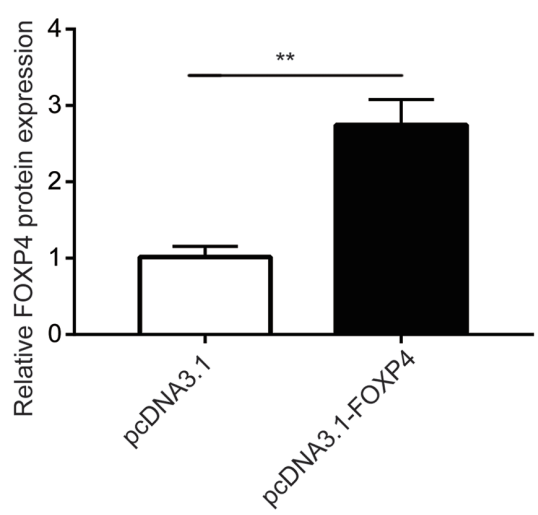

D

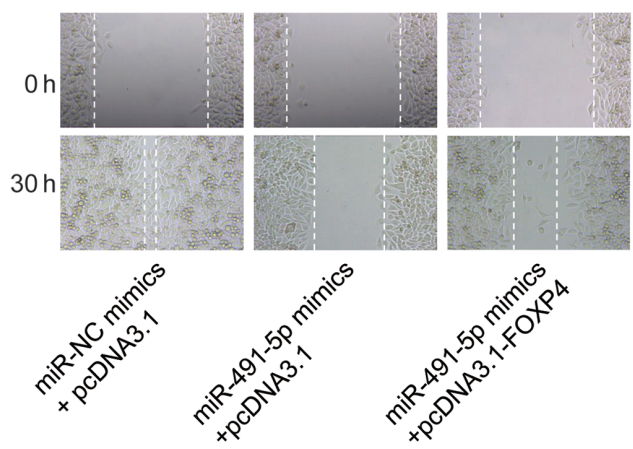

Figure 6. Overexpression of FOXP4 attenuates the miR-491-5p mimics induced inhibition on the cell proliferative and migratory abilities of A549 cells (A and B) In A549 cells, transfection of pcDNA3.1-FOXP4 increased FOXP4 protein expression levels. (C) Overexpression of FOXP4 reversed the miR-491-5p mimic induced inhibition on the proliferative ability of A549 cells. (D and E) Overexpression of FOXP4 partially reversed the miR-491-5p mimic induced inhibition of the cell migratory ability of A549 cells. ${ }^{* *} \mathrm{P}<0.01,{ }^{* * *} \mathrm{P}<0.001$. FOXP4, forkhead box P4; miR, microRNA; NC, negative control; OD, optical density.

indicating miR-491-5p as a promising target for the treatment of patients with NSCLC.

\section{Acknowledgements}

Not applicable.

\section{Funding}

No funding was received.

\section{Availability of data and materials}

The datasets used and/or analyzed during the current study are available from the corresponding author on reasonable request.

\section{Authors' contributions}

PL conceived and designed the study. FW performed the majority of the experiments and wrote the manuscript. AJ assisted with the experiments. ZZ and JL participated in the analysis and data interpretation. PL and FW confirmed the authenticity of all the raw data. All authors read and approved the final manuscript and agreed to be accountable for all aspects of the research.

\section{Ethics approval and consent to participate}

This study was performed in accordance with standard guidelines and was approved by the Ethics Committee of Yantai Laiyang Central Hospital. Written informed consent was obtained from all the patients. 


\section{Patient consent for publication}

Not applicable.

\section{Competing interests}

The authors declare that they have no competing interests.

\section{References}

1. Torre LA, Sauer AM, Chen MS Jr, Kagawa-Singer M, Jemal A and Siegel RL: Cancer statistics for Asian Americans, Native Hawaiians, and Pacific Islanders, 2016: Converging incidence in males and females. CA Cancer J Clin 66: 182-202, 2016.

2. Chen Z, Fillmore CM, Hammerman PS, Kim CF and Wong KK Non-small-cell lung cancers: A heterogeneous set of diseases. Nat Rev Cancer 14: 535-546, 2014

3. Wood SL, Pernemalm M, Crosbie PA and Whetton AD: The role of the tumor-microenvironment in lung cancer-metastasis and its relationship to potential therapeutic targets. Cancer Treat Rev 40: 558-566, 2014

4. Laskin JJ and Sandler AB: State of the art in therapy for non-small cell lung cancer. Cancer Invest 23: 427-442, 2005.

5. Lagos-Quintana M, Rauhut R, Lendeckel W and Tuschl T: Identification of novel genes coding for small expressed RNAs. Science 294: 853-858, 2001.

6. Paliouras AR, Monteverde T and Garofalo M: Oncogene-induced regulation of microRNA expression: Implications for cancer initiation, progression and therapy. Cancer Lett 421: 152-160, 2018.

7. Ye MF, Zhang JG, Guo TX and Pan XJ: MiR-504 inhibits cell proliferation and invasion by targeting LOXL2 in non small cel lung cancer. Biomed Pharmacother 97: 1289-1295, 2018.

8. Hao J, Zhao S, Zhang Y, Zhao Z, Ye R, Wen J and Li J: Emerging role of microRNAs in cancer and cancer stem cells. J Cell Biochem 115: 605-610, 2014

9. Zhou Q, Huang SX, Zhang F, Li SJ, Liu C, Xi YY, Wang L, Wang X, He QQ, Sun CC and Li DJ: MicroRNAs: A novel potential biomarker for diagnosis and therapy in patients with non-small cell lung cancer. Cell Prolif 50: e12394, 2017.

10. Xiong K, Shao LH, Zhang HQ, Jin L, Wei W, Dong Z, Zhu YQ, Wu N, Jin SZ and Xue LX: MicroRNA-9 functions as a tumor suppressor and enhances radio-sensitivity in radio-resistan A549 cells by targeting neuropilin 1. Oncol Lett 15: 2863-2870, 2018.

11. Zhang Y, Wang Y and Wang J: MicroRNA-584 inhibits cell proliferation and invasion in non-small cell lung cancer by directly targeting MTDH. Exp Ther Med 15: 2203-2211, 2018

12. Kulda V, Svaton M, Mukensnabl P, Hrda K, Dvorak P, Houdek Z, Houfkova K, Vrzakova R, Babuska V, Pesek M and Pesta M: Predictive relevance of miR-34a, miR-224 and miR-342 in patients with advanced squamous cell carcinoma of the lung undergoing palliative chemotherapy. Oncol Lett 15: 592-599, 2018

13. Zhang J, Wang T, Zhang Y, Wang H, Wu Y, Liu K and Pei C: Upregulation of serum miR-494 predicts poor prognosis in non-small cell lung cancer patients. Cancer Biomark 21: 763-768, 2018.

14. Cao Q, Mao ZD, Shi YJ, Chen Y, Sun Y, Zhang Q, Song L and Peng LP: MicroRNA-7 inhibits cell proliferation, migration and invasion in human non-small cell lung cancer cells by targeting FAK through ERK/MAPK signaling pathway. Oncotarget 7 : 77468-77481, 2016.

15. Luo J, Li H and Zhang C: MicroRNA-7 inhibits the malignant phenotypes of non-small cell lung cancer in vitro by targeting Pax6. Mol Med Rep 12: 5443-5448, 2015.

16. Mou K, Gu W, Gu C, Zhang J, Qwang W, Ren G and Tian J: Relationship between miR-7 expression and treatment outcomes with gefitinib in non-small cell lung cancer. Oncol Lett 12 4613-4617, 2016
17. Teufel A, Wong EA, Mukhopadhyay M, Malik N and Westphal H: FoxP4, a novel forkhead transcription factor. Biochim Biophys Acta 1627: 147-152, 2003.

18. Tian W, Liu J, Pei B, Wang X, Guo Y and Yuan L: Identification of miRNAs and differentially expressed genes in early phase non-small cell lung cancer. Oncol Rep 35: 2171-2176, 2016.

19. Yin Z, Ding H, He E, Chen J and Li M: Up-regulation of microRNA-491-5p suppresses cell proliferation and promotes apoptosis by targeting FOXP4 in human osteosarcoma. Cell Prolif 50: e12308, 2017.

20. Wang G, Sun Y, He Y, Ji C, Hu B and Sun Y: MicroRNA-338-3p inhibits cell proliferation in hepatocellular carcinoma by target forkhead box P4 (FOXP4). Int J Clin Exp Pathol 8: 337-344, 2015.

21. Yang T, Li H, Thakur A, Chen T, Xue J, Li D and Chen M: FOXP4 modulates tumor growth and independently associates with miR-138 in non-small cell lung cancer cells. Tumour Biol 36: 8185-8191, 2015.

22. Livak KJ and Schmittgen TD: Analysis of relative gene expression data using real-time quantitative PCR and the 2(-Delta Delta C(T)) method. Methods 25: 402-408, 2001

23. Sin $\mathrm{C}$, $\mathrm{Li} \mathrm{H}$ and Crawford DA: Transcriptional regulation by FOXP1, FOXP2, and FOXP4 dimerization. J Mol Neurosci 55: 437-448, 2015.

24. Song XL, Tang Y, Lei XH, Zhao SC and Wu ZQ: miR-618 inhibits prostate cancer migration and invasion by targeting FOXP2. J Cancer 8: 2501-2510, 2017.

25. Da C, Liu Y, Zhan Y, Liu K and Wang R: Nobiletin inhibits epithelial-mesenchymal transition of human non-small cell lung cancer cells by antagonizing the TGF- $\beta 1 / \mathrm{Smad} 3$ signaling pathway. Oncol Rep 35: 2767-2774, 2016.

26. Hui Z, Yiling C, Wenting Y, XuQun H, ChuanYi Z and Hui L: miR-491-5p functions as a tumor suppressor by targeting JMJD2B in ER $\alpha$-positive breast cancer. FEBS Lett 589: 812-821, 2015.

27. Xu Y, Hou R, Lu Q, Zhang Y, Chen L, Zheng Y and Hu B: MiR-491-5p negatively regulates cell proliferation and motility by targeting PDGFRA in prostate cancer. Am J Cancer Res 7: 2545-2553, 2017.

28. Sun R, Liu Z, Tong D, Yang Y, Guo B, Wang X, Zhao L and Huang C: miR-491-5p, mediated by Foxil, functions as a tumor suppressor by targeting Wnt3a/ $\beta$-catenin signaling in the development of gastric cancer. Cell Death Dis 8: e2714, 2017.

29. Gong F, Ren P, Zhang Y, Jiang J and Zhang $\mathrm{H}$ : MicroRNAs-491-5p suppresses cell proliferation and invasion by inhibiting IGF2BP1 in non-small cell lung cancer. Am J Transl Res 8: 485-495, 2016.

30. Sun D, Han S, Liu C, Zhou R, Sun W, Zhang Z and Qu J: Microrna-199a-5p functions as a tumor suppressor via suppressing connective tissue growth factor (CTGF) in follicular thyroid carcinoma. Med Sci Monit 22: 1210-1217, 2016.

31. Zhang Q, Li Q, Xu T, Jiang H and Xu LG: miR-491-5p suppresses cell growth and invasion by targeting Notch3 in nasopharyngeal carcinoma. Oncol Rep 35: 3541-3547, 2016.

32. Pirooz HJ, Jafari N, Rastegari M, Fathi-Roudsari M, Tasharrofi N, Shokri G, Tamadon M, Sazegar H and Kouhkan F: Functional SNP in microRNA-491-5p binding site of MMP9 3'-UTR affects cancer susceptibility. J Cell Biochem 119: 5126-5134, 2018.

33. Stetler-Stevenson WG: The role of matrix metalloproteinases in tumor invasion, metastasis, and angiogenesis. Surg Oncol Clin N Am 10: 383-392, 2001.

34. Kim S, Lee J, You D, Jeong Y, Jeon M, Yu J, Kim SW, Nam SJ and Lee JE: Berberine suppresses cell motility through downregulation of TGF- $\beta 1$ in triple negative breast cancer cells. Cell Physiol Biochem 45: 795-807, 2018.

This work is licensed under a Creative Commons Attribution-NonCommercial-NoDerivatives 4.0 International (CC BY-NC-ND 4.0) License. 